\title{
TAZ Mediates Lysophosphatidic Acid- Induced Migration and Proliferation of Epithelial Ovarian Cancer Cells
}

\author{
Geun Ok Jeong ${ }^{a, b}$ Sang Hun Shin ${ }^{a, b}$ Eun Jin Seo ${ }^{a, b}$ Yang Woo Kwon ${ }^{a, b}$ \\ Soon Chul Heo ${ }^{a, b}$ Ki-Hyung Kim ${ }^{c}$ Man-Soo Yoon ${ }^{c}$ Dong-Soo Suh ${ }^{c}$ Jae Ho Kima,b,d
}

\begin{abstract}
a Medical Research Center for Ischemic Tissue Regeneration, b Department of Physiology, 'Department of Obstetrics and Gynecology, School of Medicine, Pusan National University, Yangsan 626-870,

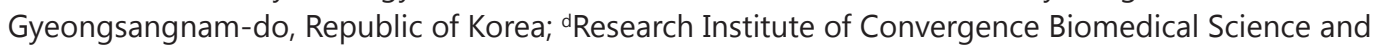
Technology, Pusan National University Yangsan Hospital, Yangsan 626-770, Gyeongsangnam-do, Republic of Korea
\end{abstract}

\section{Key Words}

Lysophosphatidic acid • TAZ • Ovarian cancer • Migration • Proliferation

\begin{abstract}
Background: Transcriptional co-activator with PDZ-binding motif (TAZ), a downstream effector of the Hippo pathway, has been reported to regulate organ size, tissue homeostasis, and tumorigenesis by acting as a transcriptional co-activator. Lysophosphatidic acid (LPA) is a bioactive lipid implicated in tumorigenesis and metastasis of ovarian cancer through activation of $G$ protein-coupled receptors. However, the involvement of TAZ in LPA-induced tumorigenesis of ovarian cancer has not been elucidated. Methods: In order to demonstrate the role of TAZ in LPA-stimulated tumorigenesis, the effects of LPA on TAZ expression and cell migration were determined by Western blotting and chemotaxis analyses in R182 human epithelial ovarian cancer cells. Results and Conclusion: Treatment of R182 cells with the LPA receptor inhibitor Ki16425 blocked LPA-induced cell migration. In addition, transfection of R182 cells with small interfering RNA specific for LPA receptor 1 resulted in abrogation of LPAstimulated cell migration. LPA induced phosphorylation of ERK and p38 MAP kinase in R182 cells and pretreatment of cells with the MEK-ERK pathway inhibitor U0126, but not the p38 MAPK inhibitor SB202190, resulted in abrogation of LPA-induced cell migration. Pretreatment of R182 cells with U0126 attenuated LPA-induced mRNA levels of TAZ and its transcriptional target genes, such as CTGF and CYR61, without affecting phosphorylation level of YAP. These results suggest that MEK-ERK pathway plays a key role in LPA-induced cell migration and mRNA expression of TAZ in R182 cells, without affecting stability of TAZ protein. In addition, small interfering RNA-mediated silencing of TAZ expression attenuated LPA-stimulated migration of R182 cells. These results suggest that TAZ plays a key role in LPA-stimulated migration of epithelial ovarian cancer cells.
\end{abstract}

Copyright (C) 2013 S. Karger AG, Base

Prof. Jae Ho Kim

and Prof. Dong-Soo Suh
Department of Physiology, School of Medicine, Pusan National University, Yangsan 626-870, P.O box number: 626-870 Gyeongsangnam-do (Republic of Korea) E-Mail jhkimst@pusan.ac.kr; E-Mail dssuh@pusan.ac.kr 


\section{Cellular Physiology and Biochemistry}

Cell Physiol Biochem 2013;32:253-263

DOI: $10.1159 / 000354434$

(1) 2013

Jeong et al.: Role of TAZ in LPA-Induced Cell Migration

\section{Introduction}

Lysophosphatidic acid (LPA) is a naturally occurring phospholipid produced by activated platelets, mesothelial cells, fibroblasts, adipocytes and some cancer cells $[1,2]$. Generation of LPA occurs through hydrolysis of phosphatidic acid by soluble phospholipase $A_{1}$ or $A_{2}[3,4]$ or hydrolysis of lysophosphatidylcholine by autotaxin/lysophospholipase D [5]. Accumulating evidence suggests that patients with ovarian cancer produce elevated levels of LPA in serum and malignant ascites [6,7]. LPA has been reported to contribute to tumorigenesis and metastasis by promoting proliferation, migration, and invasion of cancer cells [2]. LPA stimulates adhesion, migration, and invasion of ovarian cancer cells, thereby regulating tumorigenicity and aggressiveness of ovarian cancer $[8,9]$.

Specific G-protein-coupled receptors have been reported to mediate LPA-stimulated cellular responses, such as migration and proliferation of cancer cells [10]. Three of seven identified LPA receptors, Edg-2/LPA, Edg-4/LPA , and Edg-7/LPA, belong to the family of endothelial cell differentiation genes [11]. The Edg family LPA receptors, $\mathrm{LPA}_{1}, \mathrm{LPA}_{2}$ and $\mathrm{LPA}_{3}$, have been reported to show an association with tumorigenicity and aggressiveness of ovarian cancer cells $[9,12]$. Activation of LPA receptors leads to activation of various intracellular signaling pathways, including Ras-MAPK, phosphoinositide-3-kinase-Akt, phospholipase C, and RhoA [10]. The Ras-MAPK pathway has been reported to mediate LPA-stimulated migration of ovarian cancer cells [13]. However, the molecular mechanism underlying LPA-stimulated cell migration has not been elucidated.

TAZ (transcriptional co-activator with PDZ-binding motif) and its functional analog Yes-associated protein (YAP) are WW domain-containing proteins that functions as a coactivator of many transcription factors that are critical to development of various tissues [14-16]. They have been identified as a component of the emerging Hippo-LATS tumor suppressor pathway, which plays important roles in regulation of organ size control, proliferation, apoptosis, and cancer development [17-19]. The LATS tumor suppressor can phosphorylate TAZ and YAP, leading to cytoplasmic retention and functional inactivation of TAZ and YAP in mammary cells [20]. TAZ and YAP act mainly through TEAD family transcription factors to stimulate expression of its downstream targets connective tissue growth factor (CTGF) and Cyr61 that promote proliferation and inhibit apoptosis [21]. In addition, TAZ is overexpressed in invasive breast cancer tissues [22], and overexpression of TAZ has been shown to enhance proliferation, migration, transformation, and epithelial-tomesenchymal transition in immortalized mammary epithelial cells [20,22]. LPA has recently been reported to inhibit the Hippo pathway kinases LATS1/2 through the $G_{12 / 13}$-mediated pathway, thereby activating TAZ and YAP [23]. These results suggest a possible implication of TAZ in LPA-induced cellular responses, including migration of cancer cells. However, it is still unclear whether TAZ plays a role in LPA-induced migration of ovarian cancer cells. In the current study, we demonstrate an important role of TAZ in LPA-stimulated migration of ovarian cancer cells and characterize the signaling mechanisms associated with LPAinduced expression of TAZ.

\section{Materials and Methods}

\section{Materials}

RPMI 1640 medium was purchased from HyClone (Thermo scientific, Utah). Phosphate-buffered saline, trypsin, and Lipofectamin reagent were purchased from Invitrogen (Carlsbad, CA). LPA (1-oleoyl-2hydroxy-sn-glycero-3-phosphate) and Ki16425 were purchased from Sigma-Aldrich (St. Louis, MO). Antibodies against TAZ, YAP, p-YAP, ERK, p-ERK, p38, and p-p38 were purchased from Cell Signaling Technology, Inc. (Danvers, MA). Anti-glyceraldehyde-3-phosphate dehydrogenase (GAPDH) antibody was purchased from EMD Millipore (Billerica, MA). 


\section{Cellular Physiology and Biochemistry}

Cell Physiol Biochem 2013;32:253-263

DOI: $10.1159 / 000354434$

Publisned onine: July 31, 2013

(c) 2013 S. Karger AG, Basel

www.karger.com/cpb

Jeong et al.: Role of TAZ in LPA-Induced Cell Migration

Cell culture

R182 and SKOV3 cells were maintained in RPMI 1640 medium containing 10\% fetal bovine serum and penicillin/streptomycin at $37^{\circ} \mathrm{C}$ with $5 \% \mathrm{CO}_{2}$ R182 cells were kindly donated by Prof. Gil Mor (Yale University School of Medicine). SKOV3 cells were purchased from the American Type Culture Collection (Manassas, VA). To determine the effects of LPA on TAZ expression, cells were seeded onto 6-well plates, and incubated in serum-starved RPMI 1640 medium for $24 \mathrm{~h}$, followed by treatment with LPA or vehicles $(0.1 \%$ fatty acid-free bovine serum albumin).

\section{Transfection of small interfering RNA (siRNA)}

For siRNA experiments, R182 cells were trypsinized and suspended in OPTI-MEM at a density of $5 \times$ $10^{4} \mathrm{cells} / \mathrm{mL}$ and then added to each well of the plates. Cells were transfected with appropriate siRNAs using Lipofectamine reagent according to the manufacturer's instructions (Invitrogen). siRNA duplexes were synthesized, desalted, and purified by Samchully Pharm. Co. Ltd. (Siheung, Gyeonggi, Korea), as follows: LPA ${ }_{1}, 5^{\prime}$-GGACUUGGAAUCACUGUUUUU-3' (sense) and 5'-AAACAGUGAUUCCAAGUCCUU-3' (antisense); TAZ, 5'-AGGAACAAACGUUGACUUATT-3' (sense) 5'-UAAGUCAACGUUUGUUCCUTT -3' (anti-sense). The control siRNA (D-001206-13-05) was purchased from Dharmacon, Inc. (Chicago, IL).

\section{Western blotting}

Western blot was performed according to standard procedure using SDS-PAGE. Cells were lysed using lysis buffer (20 mM Tris-HCl, 1 mM EGTA, 1 mM EDTA, $10 \mathrm{mM} \mathrm{NaCl}, 0.1 \mathrm{mM}$ phenylmethylsulfonyl fluoride, $1 \mathrm{mM} \mathrm{Na}_{3} \mathrm{VO}_{4}, 30 \mathrm{mM}$ sodium pyrophosphate, $25 \mathrm{mM} \beta$-glycerol phosphate, $1 \%$ Triton X-100, pH 7.4). Cell lysates were centrifuged for $15 \mathrm{~min}$ at $4^{\circ} \mathrm{C}$, and supernatants were used for Western blotting. Lysates were resolved by SDS-PAGE, transferred onto a nitrocellulose membrane (GE Healthcare Life Sciences, Piscataway, $\mathrm{NJ}$ ), and then stained with $0.1 \%$ Ponceau S solution (Sigma-Aldrich) to ensure equal loading. After blocking with $5 \%$ nonfat milk for $30 \mathrm{~min}$, the membranes were immunoblotted with various antibodies overnight at $4{ }^{\circ} \mathrm{C}$, and the bound antibodies were visualized with horseradish peroxidase-conjugated secondary antibodies using the enhanced chemiluminescence Western blotting system (GE Healthcare Life Sciences).

Reverse transcription-polymerase chain reaction (RT-PCR)

All RNA samples were prepared using TRizol reagent (Sigma-Aldrich). RNA samples (2 $\mu \mathrm{g}$ ) were reverse-transcribed to complementary DNA using M-MLV Reverse transcriptase (Promega) and $0.5 \mu \mathrm{g}$ of oligo (dT) 15 primer (Promega). The cDNA in $1 \mu \mathrm{L}$ of the reaction mixture was amplified with $0.5 \mathrm{U}$ of GoTaq DNA polymerase, $25 \mu \mathrm{M} \mathrm{MgCl}_{2} 10 \mu \mathrm{M}$ dNTP (Promega), and 10 pmol each of sense and antisense primers. The thermal cycle profile was as follows: denaturation at $95^{\circ} \mathrm{C}$ for $30 \mathrm{~s}$, annealing at $51-55^{\circ} \mathrm{C}$ for 30 $\mathrm{s}$ depending on the primers used, and extension at $72^{\circ} \mathrm{C}$ for $90 \mathrm{~s}$. Each PCR reaction was carried out for 25 30 cycles, and PCR products were size fractionated on 1.4\% ethidium bromide/agarose gel and quantified under UV transillumination.

Real-time PCR assay was performed in 7500 real-time PCR machine (Applied Biosystems) and the data generated were analyzed with the Applied biosystems 7500 Software (version 2.0.6). The cDNA was diluted and used for quantification (with $\beta$-actin gene as a control) by real-time PCR. The assay was carried out in a total reaction volume of $20 \mu \mathrm{l}$ that consisted of $10 \mu \mathrm{l}$ of 2X SYBR Green Master Mix(2X) (Applied Biosystems), 10 picomoles of each primer (COSMO genetech) and $2 \mu \mathrm{l}$ of each dilute cDNA. The protocol was as follows: 40 cycles at $95^{\circ} \mathrm{C}$ for $15 \mathrm{~s}$ and $60^{\circ} \mathrm{C}$ for $1 \mathrm{~min}$. The primer pairs are as follows in this study : LPA receptor 5'-TCT TCT GGG CCA TTT TCA AC-3', 5'-TGC CTR AAG GTG GCG CTC AT-3', LPA 2 receptor 5' -CCT ACC TCT TCC TCA TGT TC-3', 5'-TAA AGG GTG GAG TCC ATC AG-3'; LPA receptor 5'-GGA ATT GCC TCT GCA ACA TCT-3', 5'-GTC TTT ACT CCT TGG AGG CCA TGT-3'; TAZ 5'-CAG CAA TGT GGA TGA GAT GG-3', 5'-TCA TTG AAG AGG GGG ATC AG-3': GAP DH 5'-TCC ATG ACA ACT TTG GTA TCG-3', $5^{\prime}$-TGT AGC CAA ATT CGT TGT CA-3'; $\beta$-actin $5^{\prime}$-AAC ACC CCA GCC ATG TAC G-3' 5' $^{\prime}$-ATG TCA CGC ACG ATT TCC C-3', CTGF 5'-TAT GAT TAG AGC CAA CTG CC-3', 5'-GAG TAC GGA TGC ACT TTT TG-3'; CYR61 5'-TGG AGT TCA CGA GAA ACA AT-3', 5'-GTA ACT CGT GTG GAG ATA CC-3'.

Immunostaining and analysis of images

R182 cells were seeded on coverslips in a 24-well plate. After treatment with LPA, cells were fixed with $4 \%$ paraformaldehyde for $10 \mathrm{~min}$, and washed twice with phosphate-buffered saline, followed by 


\section{Cellular Physiology and Biochemistry}

Cell Physiol Biochem 2013;32:253-263

DOI: $10.1159 / 000354434$

Publisned oniIne: July 3I, 2013

(c) 2013 S. Karger AG, Basel

www.karger.com/cpb

Jeong et al.: Role of TAZ in LPA-Induced Cell Migration

permeabilization with $0.1 \%$ Triton X-100 in TBS. After blocking in 5\% BSA for $1 \mathrm{~h}$ at room temperature, cells were incubated with anti-TAZ primary antibody overnight at $4{ }^{\circ} \mathrm{C}$. After washing with phosphate-buffered saline, the specimens were incubated with Alexa 488 goat anti-rabbit secondary antibody, followed by washing and mounting in Vectashield medium (Vector Laboratories) with 4',6-diamidino-2-phenylindole (DAPI) for visualization of nuclei. A laser scanning confocal microscopy (Olympus FluoView FV1000) was used for visualization of stained specimens.

Cell migration and scratch wound healing assay

Cell migration assay was performed using a chemotaxis assay and a scratch wound healing assay. For measurement of chemotactic cell migration, cells were harvested with $0.05 \%$ trypsin containing $0.02 \%$ EDTA, washed once, and suspended in serum-free media at a concentration of $1 \times 10^{5} \mathrm{cells} / \mathrm{ml}$. A membrane filter with 8- $\mu \mathrm{m}$ pores of the disposable 96-well chemotaxis chamber (Neuro Probe, Inc., Gaithersburg, MD) was pre-coated for $4 \mathrm{~h}$ with $20 \mu \mathrm{g} / \mathrm{mL}$ rat-tail collagen at room temperature; an aliquot (50 $\mu \mathrm{L}$ ) of cell suspension was loaded into the upper chamber, and test reagents were then placed in the lower chamber, unless otherwise specified. For elucidation of signaling pathways involved in LPA-induced migration, the cells were pre-incubated with pharmacological inhibitors for $10 \mathrm{~min}$ before loading. After incubation of the cells with LPA in the absence or presence of inhibitors for $12 \mathrm{~h}$ at $37^{\circ} \mathrm{C}$, the upper surface of each filter was scraped free of cells by wiping it with a cotton swab, and the filters were then dis-assembled. The number of cells that had migrated to the lower surface of each filter was determined by counting the cells in three places under microscopy at $\times 100$ magnification after staining with Hoechst.

For measurement of cell migration using a scratch wound healing assay, cells were seeded in 24-well plates at a density of $5 \times 10^{4}$ and grown to confluence, followed by serum starvation for $24 \mathrm{~h}$. The serumstarved cells were scratched once per well using a P20 pipette tip for creation of an artificial wound and treated with serum-free medium in the absence or presence of LPA. After $12 \mathrm{~h}$, wounds were photographed using a digital camera mounted on an inverted microscope.

\section{Cell proliferation assay}

To measure the effects of LPA on cell proliferation of R182 cells, a colorimetric 3-(4,5-dimethylthiazol2-yl)-2,5-diphenyltetrazolium bromide (MTT) assay was used: MTT is metabolized by NAD-dependent dehydrogenase to form a colored reaction product (formazan), and the amount of dye formed correlates directly with the number of cells. For determination of cell numbers, R182 cells were seeded in a 24-well culture plate at a density of $2 \times 10^{4}$ cells/well, cultured for $48 \mathrm{~h}$ in normal growth medium, serum-starved for $24 \mathrm{~h}$, and treated with various reagents (or a vehicle control) for the indicated times. Cells were washed twice with PBS and incubated with $100 \mu \mathrm{l}$ of MTT $(0.5 \mathrm{mg} / \mathrm{ml})$ for $2 \mathrm{~h}$ at $37^{\circ} \mathrm{C}$. Formazan granules generated by the cells were dissolved in $100 \mu \mathrm{l}$ of dimethylsulfoxide, and the absorbance of the solution at $562 \mathrm{~nm}$ was determined using a PowerWave ${ }_{\mathrm{x}}$ microplate spectrophotometer (Bio-Tek Instruments, Inc.; Winooski, VT) after dilution to a linear range.

\section{Statistics}

The results of multiple observations are presented as mean \pm S.D. Student's $t$ test was used for analysis of differences between the two groups. For multivariate data analysis, two-way ANOVA was used for assessment of group differences, followed by post hoc comparisons tested using Scheffe's method.

\section{Results}

LPA treatment induces expression of TAZ in R182 epithelial ovarian cancer cells

To explore the effect of LPA on expression of TAZ in ovarian cancer cells, two different epithelial ovarian cancer cell lines, R182 and SKOV3 cells, were treated with LPA for increasing periods of time. Expression of TAZ showed a time-dependent increase upon treatment with LPA (Fig. 1A). Increased expression of TAZ was detected at $3 \mathrm{~h}$ after treatment with LPA in R182 cells and maximal stimulation was observed at $6 \mathrm{~h}$. LPA-induced expression of TAZ was more evident in R182 cells than in SKOV3 cells. LPA treatment caused a mobility shift of TAZ, suggesting dephosphorylation of TAZ in response to LPA. To explore the effect of 
Fig. 1. Effects of LPA on expression and localization of TAZ in epithelial ovarian cancer cells. (A) Time dependence of LPA-induced expression of TAZ. R182 and SKOV3 cells were treated with $1 \mu \mathrm{M}$ LPA or vehicles for the indicated time periods. (B) Quantification of TAZ expression and YAP phosphorylation. Densities of TAZ and p-YAP from panel (A) were quantified and normalized to those of GAPDH and YAP, respectively. Data indicate mean \pm SD $(\mathrm{n}=4) . *, \mathrm{p}<0.05$. (C) R182 cells were treated with increasing concentrations of LPA for $6 \mathrm{~h}$. Expression levels of TAZ and GADPH were determined by Western blotting. (D) Nuclear localization of TAZ in LPA-treated cells. R182 cells were treated with $1 \mu \mathrm{M}$ LPA or vehicles (control) for $6 \mathrm{~h}$, followed by immunostaining with anti-TAZ

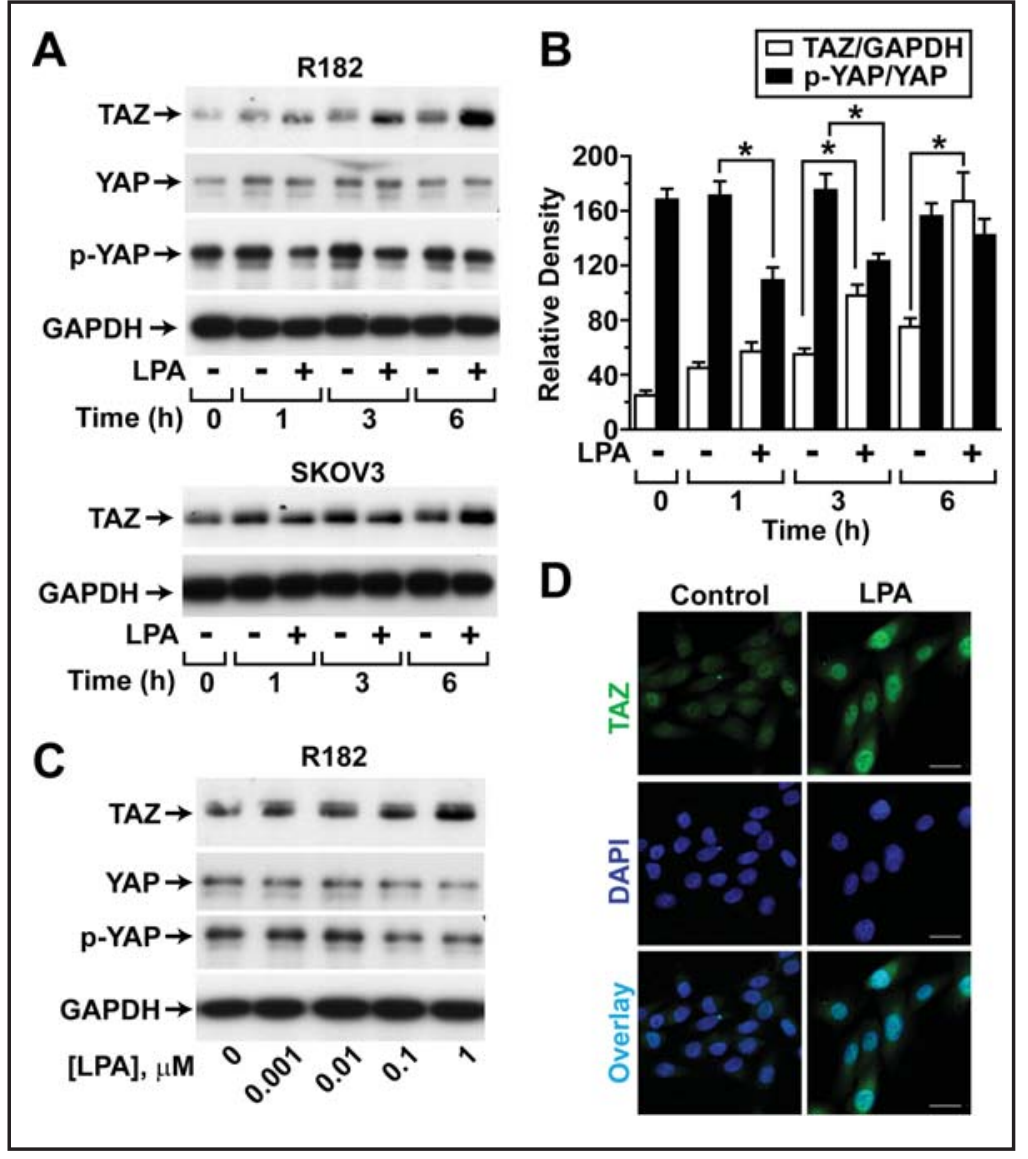
antibody. Overlaid images of TAZ (green color) and DAPI (nucleus, blue color) are shown. Representative data from three independent experiments are shown. Bar $=30 \mu \mathrm{m}$.

LPA on dephosphorylation of TAZ/YAP, phosphorylation levels of YAP was determined by Western blotting using a phospho-YAP antibody (S127). LPA treatment resulted in a rapid decrease in YAP phosphorylation (Fig. 1A). LPA-induced dephosphorylation of YAP was detected as early as $1 \mathrm{~h}$ and recovered $6 \mathrm{~h}$ after LPA treatment. These results suggest that LPA induces dephosphorylation of TAZ and YAP in R182 cells, while increased protein levels of YAP were hardly observed by LPA treatment. In addition, treatment with LPA resulted in a dose-dependent increase in TAZ expression along with decrease of YAP phosphorylation (Fig. 1C). Immunostaining with anti-TAZ antibody showed LPA-stimulated expression of TAZ, which was mostly localized in nuclei of R182 cells (Fig. 1D), suggesting LPA-induced TAZ expression in the nuclear fraction of epithelial ovarian cancer cells.

\section{LPA stimulates migration of R182 cells through a LPA - dependent mechanism}

To explore the role of LPA in migration of R182 cells, chemotactic migration was determined using a Boyden chamber apparatus. As shown in Fig. 2A, treatment with LPA resulted in stimulation of chemotactic migration of R182 cells in a dose-dependent manner. To clarify involvement of LPA receptors in LPA-induced cell migration, the effect of Ki16425, an inhibitor specific for LPA receptors 1 and 3, on LPA-stimulated migration was explored. Pretreatment of cells with Ki16425 resulted in complete abrogation of LPAstimulated migration of R182 cells (Fig. 2B), suggesting involvement of LPA or $^{2} \mathrm{LA}_{3}$ in the LPA-stimulated cell migration. Because $\mathrm{LPA}_{1}$ and $\mathrm{LPA}_{2}$, but not $\mathrm{LPA}_{3}$, are expressed in R182 cells (Fig. 2C), we next examined the involvement of LPA in LPA-induced cell migration. In R182 cells, expression of LPA in R182 cells was silenced by transfection with LPA -specific $_{1}$ 
Fig. 2. Role of $\mathrm{LPA}_{1}$ in LPA-induced migration of ovarian cancer cells. (A) Dose-dependent effect of LPA on chemotactic migration of R182 cells. Data indicate mean \pm SD $(n=4) .{ }^{*}, \mathrm{p}<0.05$ vs control. (B) mRNA expression of three LPA receptors (LPA1, LPA2, and LPA3) and GAPDH. (C) Effects of Ki16425 on LPA-stimulated migration of R182 cells. LPAinduced migration of R182 cells was determined in the absence or presence of $1 \mu \mathrm{M}$ Ki16425. (D) siRNA-mediated silencing of LPA1. R182 cells were transfected with $\mathrm{LPA}_{1}$-specific siRNA (si-LPA $)_{1}$ or control siRNA (si-control), and expression levels of LPA1 and GAPDH were determined by RT-PCR. (E) siRNA-transfected R182 cells were treated with $1 \mu \mathrm{M}$ LPA or vehicles for $12 \mathrm{~h}$, followed by determination of cell migration. Data indicate mean \pm SD $(n=4)$. $*, \mathrm{p}<0.05$.

Fig. 3. Role of LPA1 in LPA-induced expression of TAZ. (A) Effect of Ki16425 on LPA-induced expression of TAZ. R182 cells were treated with 1 $\mu \mathrm{M}$ LPA or vehicles in the absence or presence of 1 $\mu \mathrm{M}$ Ki16425 for 6 h. (B) R182 cells transfected with si-control or si-LPA1 were treated with $1 \mu \mathrm{M}$ LPA or vehicles for $6 \mathrm{~h}$. Expression levels of TAZ and GAPDH were determined by Western blot analysis. Representative data from three independent experiments are shown.
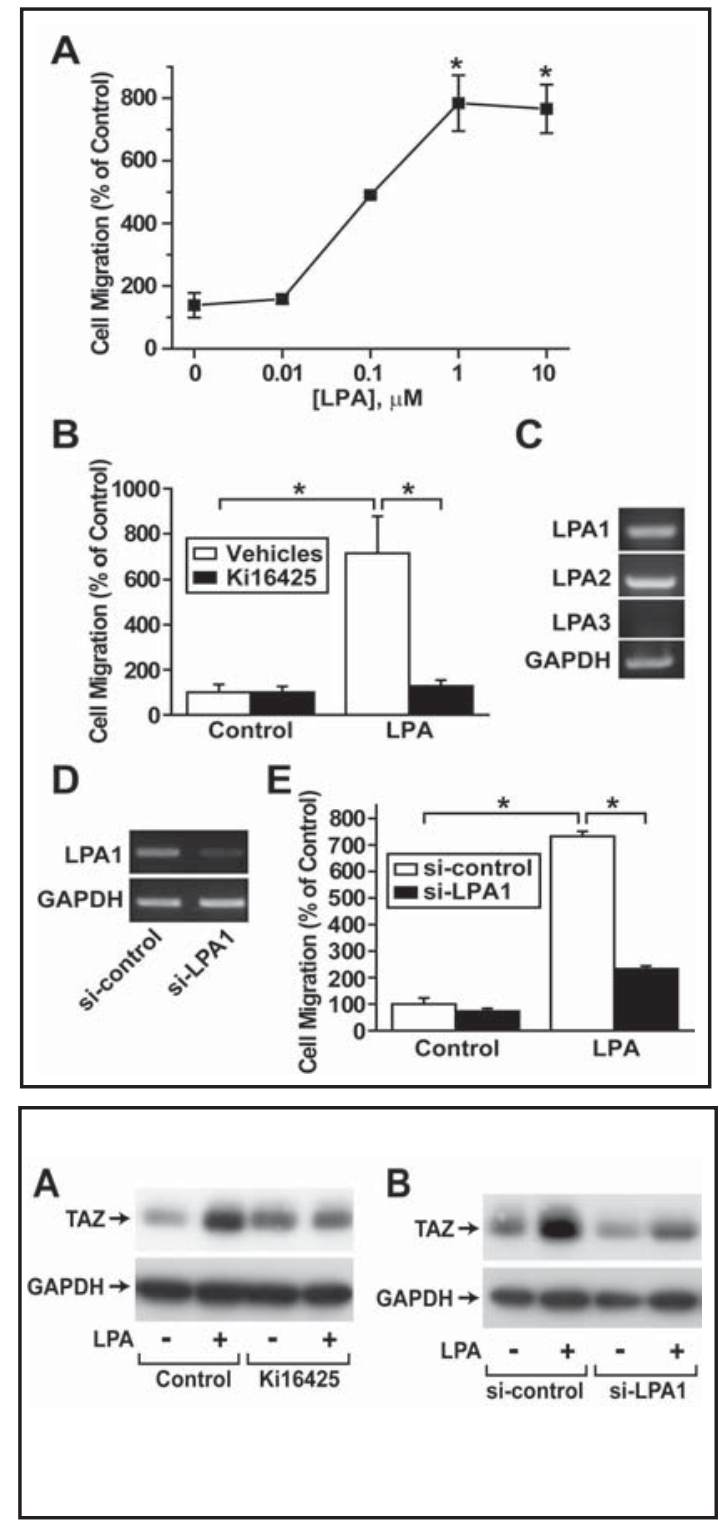

siRNA (Fig. 2D). siRNA-mediated silencing of LPA 1 expression markedly inhibited LPAstimulated migration of R182 cells (Fig. 2E). These results suggest a critical role of LPA in LPA-simulated migration of epithelial ovarian cancer cells.

$L P A_{1}$ is involved in LPA-induced expression of TAZ

To explore the question of whether LPA 1 is involved in LPA-induced expression of TAZ, the effect of Ki16425 on LPA-stimulated expression of TAZ was determined. As shown in Fig. 3A, LPA-stimulated expression of TAZ in R182 cells was inhibited by pretreatment of cells with Ki16425. In addition, siRNA-mediated silencing of LPA resulted in attenuation of LPA-induced expression of TAZ (Fig. 3B). These results suggest a key role of LPA in not only LPA-induced cell migration but also TAZ expression.

ERK is involved in LPA-induced cell migration and TAZ expression

LPA-induced stimulation of LPA, leads to activation of MAP kinases, including ERK, JNK, and p38 MAPK [10]. To clarify the involvement of MAP kinases in LPA-stimulated chemotaxis and TAZ expression in R182 cells, we explored the effects of LPA on phosphorylation of MAP 
Fig. 4. Role of MAP kinases in LPA-induced cell migration and TAZ expression. (A) LPAinduced phosphorylation of MAP kinases. R182 cells were treated with $1 \mu \mathrm{M}$ LPA for the indicated time periods, and phosphorylation and expression levels of ERK and p38 MAPK were determined by Western blotting. (B) Effects of MAPK inhibitors on LPA-induced cell migration. LPA-induced migration of R182 cells was determined in the absence or presence of $10 \mu \mathrm{M}$ U0126, SB202190, or Y27632. Data indicate mean \pm SD $(\mathrm{n}=4) . *, \mathrm{p}<0.05$. (C) Inhibitory effect of U0126 on LPA-induced phosphorylation of ERK. R182 cells were treated with $1 \mu \mathrm{M}$ LPA for the indicated times in the absence or presence of U0126 or vehicles $(0.1 \%$ DMSO). Phosphorylation and expression levels of ERK

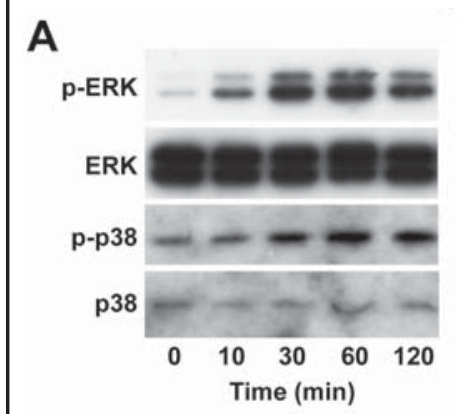

B
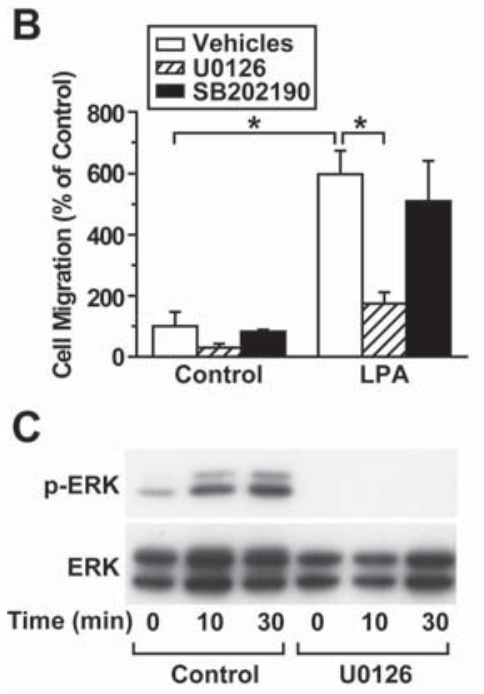

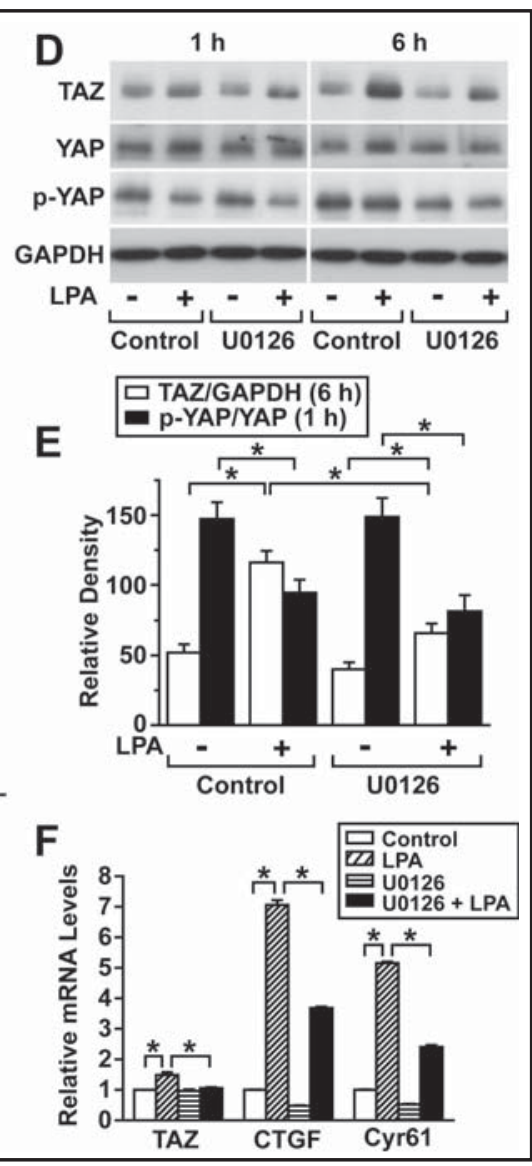

were determined by Western blot analysis. (D) Effects of U0126 on LPA-induced TAZ expression and YAP dephosphorylation. R182 cells were treated with $1 \mu \mathrm{M}$ LPA or vehicles in the absence or presence of $10 \mu \mathrm{M}$ U0126 for $1 \mathrm{~h}$ or $6 \mathrm{~h}$, followed by Western blotting of TAZ, YAP, p-YAP, and GAPDH. Representative data from four independent experiments are shown. (E) Relative Densities of TAZ and p-YAP were quantified at $6 \mathrm{~h}$ and $1 \mathrm{~h}$, respectively, and normalized to those of GAPDH and YAP. Data indicate mean \pm SD $(n=4) .{ }^{*}, \mathrm{p}<0.05$. (F) Effect of U0126 on LPA-stimulated mRNA levels of TAZ, CTGF, and Cyr61. R182 cells were treated with 1 $\mu \mathrm{M}$ LPA or vehicles in the absence or presence of $10 \mu \mathrm{M}$ U0126 for $1 \mathrm{~h}$, followed by determination of mRNA levels of TAZ, CTGF, and Cyr61 by real-time RT-PCR and normalization to the levels of $\beta$-actin in each sample. Data indicate mean \pm SD $(\mathrm{n}=3)$. * $\mathrm{p}<0.05$.

kinases. As shown in Fig. 4A, treatment with LPA induced phosphorylation of ERK and p38 MAPK in a time-dependent manner. However, phosphorylation of JNK was not detected after treatment with LPA in R182 cells (data not shown). To explore involvement of ERK and p38 MAPK in LPA-stimulated cell migration, we examined the effects of the ERK inhibitor U0126 and the p38 MAPK inhibitor SB202190 on LPA-induced cell migration. Pretreatment of cells with U0126, but not SB201290, markedly inhibited LPA-induced migration of R182 cells (Fig. 4B), suggesting involvement of ERK in LPA-stimulated cell migration.

To assess the role of ERK in LPA-induced expression of TAZ, we next examined the effect of U0126 on LPA-stimulated phosphorylation of ERK and expression of TAZ. As shown in Fig. 4C, pretreatment of R182 cells with U0126 resulted in blockade of LPAinduced phosphorylation of ERK. In addition, LPA stimulation resulted in the increase of TAZ expression at $6 \mathrm{~h}$, and pretreatment of R182 cells with U0126 markedly but not completely attenuated LPA-induced protein levels of TAZ (Fig. 4D and 4E). YAP phosphorylation was decreased at $1 \mathrm{~h}$ after LPA treatment; however, pretreatment with U0126 did not affect the LPA-induced dephosphorylation of YAP and mobility shift of TAZ. These results suggest 
Fig. 5. Role of TAZ in LPA-induced migration of epithelial ovarian cancer cells. (A) siRNA-mediated silencing of LPA-induced expression of TAZ. R182 cells were transfected with si-control or si-TAZ, and the expression levels of TAZ and GAPDH were determined by Western blotting. (B) Inhibition of LPA-induced chemotactic migration by silencing of TAZ expression. R182 cells were transfected with si-control or si$\mathrm{TAZ}$, and then treated with $1 \mu \mathrm{M} \mathrm{LPA}$ or vehicles (control) for $12 \mathrm{~h}$, followed by determination of chemotactic migration. Data indicate mean \pm SD $(\mathrm{n}=4) .{ }^{*}, \mathrm{p}<0.05$. (C) Role of TAZ in LPAstimulated healing of wound scratch.

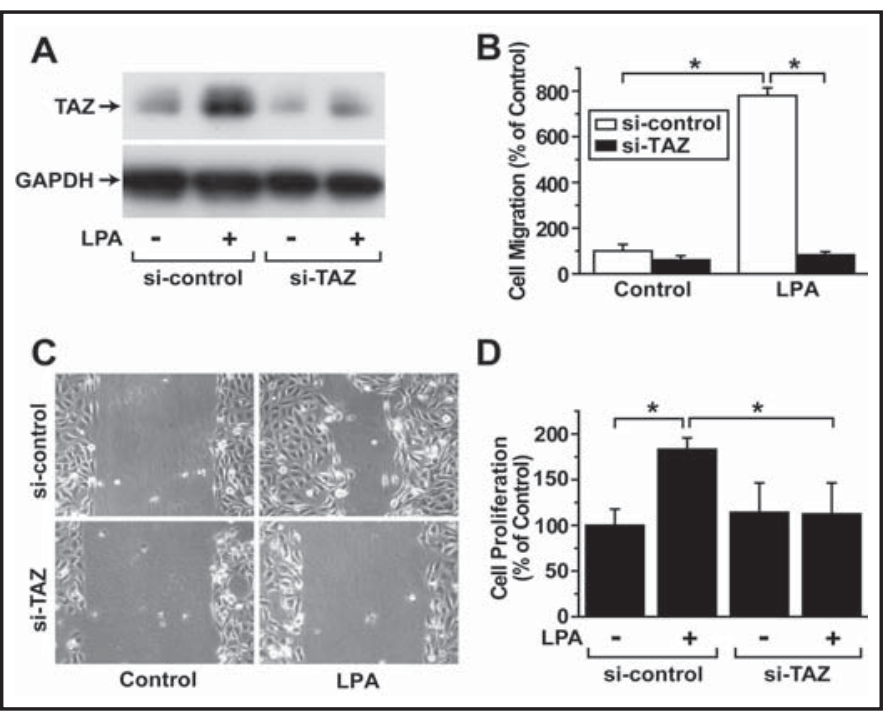
Wounds were generated in siRNA-transfected R182 cells, treated with $1 \mu \mathrm{M}$ LPA or vehicles (control) for 12 $\mathrm{h}$, and then wound healing was photographed using a digital camera mounted on an inverted microscope. (D) Inhibition of LPA-induced proliferation by silencing of TAZ expression. siRNA-transfected R182 cells were treated with $1 \mu \mathrm{M}$ LPA or vehicles (control) for 3 days, followed by determination of cell proliferation. Data indicate mean $\pm \operatorname{SD}(\mathrm{n}=4)$. ${ }^{*}, \mathrm{p}<0.05$.

that ERK plays a key role in LPA-induced expression of TAZ in R182 cells without affecting phosphorylation of YAP and TAZ.

TAZ and YAP act as a co-factor for TEAD transcription factors to stimulate gene expression of proliferation-proliferating genes such as CTGF and Cyr61. To explore whether LPA treatment can induce gene expression of TAZ and its target genes CTGF and Cyr61, we quantified the mRNA levels of TAZ, CTGF, and Cyr61. As shown in Fig. 4F, mRNA levels of TAZ, CTGF, and Cyr61 were increased in response to LPA treatment. To determine the role of ERK in LPA-induced expression of TAZ, CTGF, and Cyr61, we examined the effects of U0126 on mRNA levels of the genes. Treatment with U0126 abrogated LPA-induced mRNA expression of TAZ; however, LPA-induced mRNA expression of CTGF and Cyr61 were markedly, but not completely, attenuated by U0126 treatment. These results suggest that ERK-dependent transcription of TAZ plays a crucial role in the LPA-induced transcription of CTGF and Cyr61.

TAZ plays a key role in LPA-stimulated migration of R182 ovarian cancer

To explore the role of TAZ in migration of ovarian cancer cells, we examined the effects of siRNA-mediated silencing of TAZ on LPA-induced migration of R182 cells. LPA-stimulated expression of TAZ was blocked by transfection of R182 cells with TAZ-specific siRNA (Fig. 5A). To determine whether TAZ is involved in LPA-stimulated cell migration, we next examined the effect of siRNA-mediated silencing of TAZ on LPA-induced cell migration. As shown in Fig. 5B, LPA-stimulated migration of R182 cells was blocked by transfection of cells with TAZ-specific siRNA. To confirm this finding, we next examined involvement of TAZ in LPAstimulated motility of ovarian cancer cells in a scratch wound healing assay. As shown in Fig. 5C, treatment with LPA enhanced motility of R182 cells and wound healing, and silencing of TAZ expression by transfection with TAZ-specific siRNA resulted in abrogation of LPAstimulated wound healing.

LPA reportedly stimulates not only cell migration but also proliferation in various cell types [2]. In order to explore whether TAZ is involved in regulation of LPA-stimulated proliferation of epithelial ovarian cancer cells, we examined the effect of siRNA-mediated silencing of TAZ expression on LPA-induced cell proliferation. Treatment of R182 cells with 


\section{Cellular Physiology and Biochemistry}

Cell Physiol Biochem 2013;32:253-263

DOI: 10.1159/000354434

Jeong et al.: Role of TAZ in LPA-Induced Cell Migration

LPA-induced cell proliferation, and siRNA-mediated silencing of TAZ expression abrogated LPA-induced proliferation (Fig. 5D). Taken together, these results indicate that TAZ plays a key role in LPA-induced migration and proliferation of R182 cells.

\section{Discussion}

In the current study, we demonstrated that LPA stimulates TAZ expression in two different epithelial ovarian cancer cell lines, R182 and SKOV3 cells. In addition, treatment with LPA induced nuclear localization of TAZ and a rapid and transient dephosphorylation of YAP in R182 cells. It has been reported that not only LPA but also sphingosine-1-phosphate activates YAP and TAZ through $\mathrm{G}_{12 / 13}$-RhoA-dependent inhibition of the Hippo pathway kinases LATS1/2 [23]. Treatment with S1P induced nuclear localization of YAP through S1P receptor, a $G$ protein-coupled receptor $[23,24]$. In addition, protease-activated receptors decrease phosphorylation and increase nuclear localization of YAP/TAZ by inhibiting LATS1/2 through a $\mathrm{G}_{12 / 13}$-dependent mechanism [25]. These results support the pivotal role of $\mathrm{G}$ protein-coupled receptors, including LPA, in regulation of the Hippo-YAP/TAZ pathway.

We demonstrated a pivotal role of ERK in LPA-induced migration of R182 cells. In support of the current study, LPA has been reported to induce migration of pancreatic carcinoma cells through a $\mathrm{G}_{\mathrm{i} / \mathrm{o}}$-ERK-dependent pathway [26]. In addition, LPA stimulated migration of MDA-MB-231 breast cancer cells through a phosphoinositide-3-kinase-ERK-dependent mechanism [27]. It has been reported that JNK cooperates with oncogenic Ras to inactivate the Hippo pathway, leading to tumorigenesis [28]. Raf-1 kinase inhibits MST2/Hippo kinase by preventing its dimerization and phosphorylation of MST2 through a mechanism independent of the kinase activity of Raf-1 [29]. In the current study, we demonstrated that LPA-induced mRNA level of TAZ gene was abrogated by pretreatment with the MEK1/2ERK pathway inhibitor U0126, implying a crucial role of ERK in LPA-induced transcription of TAZ gene. However, LPA-stimulated protein level of TAZ was partially inhibited by U0126 treatment. Furthermore, mRNA expression of TAZ target genes, CTGF and Cyr61, was partially attenuated by pretreatment with U0126. We showed here that LPA-induced dephosphorylation of YAP and mobility shift of TAZ were not abrogated by U0126 treatment, suggesting that ERK is not involved in LPA-induced regulation of protein stability of TAZ and YAP. Increased stability of TAZ protein has been reported to be responsible for LPA-induced expression of TAZ [23]. Taken together, these results suggest that not only transcription of TAZ gene but also stabilization of TAZ protein is implicated in LPA-induced increase of TAZ protein in R182 cells, although the molecular mechanism by which ERK activation leads to up-regulated expression of TAZ and its target genes needs to be clarified further.

siRNA-mediated knockdown of TAZ expression resulted in abrogation of LPA-induced migration and proliferation of R182 ovarian cancer cells. siRNA-mediated silencing of TAZ expression has been shown to down-regulate proliferation and migration of immortalized mammary epithelial cells [22]. It has been recently reported that LPA treatment induced migration of human epithelial ovarian cancer cells through an YAP-dependent mechanism [30]. The role of Hippo signaling in regulation of cell migration was supported by a recent report suggesting that Hippo/MST1 kinase mediates chemotaxis through regulation of spreading and cell adhesion [31]. An association of high levels of nuclear YAP in primary tumor tissues with poor survival in ovarian cancer patients has been reported [32]. In addition, overexpression of YAP resulted in increased cell proliferation and migration, resistance to cisplatin-induced apoptosis, and anchorage-independent growth in immortalized ovarian surface epithelial cells, suggesting the role of Hippo/YAP as an ovarian cancer oncogene [32]. Taken together, these results suggest a pivotal role of TAZ in regulation of migration, invasion, and metastasis of ovarian cancer. Although further exploration of the molecular mechanism by which TAZ regulates LPA-induced cell migration is needed, in the present study, we report on the LPA - TAZ signaling cascade as a novel target for treatment of ovarian cancer. 


\section{Cellular Physiology and Biochemistry}

Cell Physiol Biochem 2013;32:253-263

Jeong et al:: Role of TAZ in LPA-Induced Cell Migration

\section{Abbreviations}

GAPDH (glyceraldehydes-3-phosphate dehydrogenase); LPA (lysophosphatidic acid); $L_{1}$ (LPA receptor 1); $L_{2} A_{2}$ (LPA receptor 2); $L^{2} A_{3}$ (LPA receptor 3); RT-PCR (reverse transcription-polymerase chain reaction); siRNA (small interfering RNA); TAZ (Transcriptional coactivator with PDZ-binding motif); YAP (Yes-associated protein).

\section{Acknowledgements}

This research was supported by programs through the National Research Foundation of Korea (NRF) funded by the Ministry of Education, Science and Technology (2010-0011288) and Medical Research Institute Grant (2009-37), Pusan National University.

\section{References}

1 Aoki J: Mechanisms of lysophosphatidic acid production. Semin Cell Dev Biol 2004;15:477-489.

-2 Mills GB, Moolenaar WH: The emerging role of lysophosphatidic acid in cancer. Nat Rev Cancer 2003;3:582-591.

-3 Moolenaar WH: Lysophosphatidic acid, a multifunctional phospholipid messenger. J Biol Chem 1995;270:12949-12952.

4 Sano T, Baker D, Virag T, Wada A, Yatomi Y, Kobayashi T, Igarashi Y, Tigyi G: Multiple mechanisms linked to platelet activation result in lysophosphatidic acid and sphingosine 1-phosphate generation in blood. J Biol Chem 2002;277:21197-21206.

-5 Umezu-Goto M, Kishi Y, Taira A, Hama K, Dohmae N, Takio K, Yamori T, Mills GB, Inoue K, Aoki J, Arai H: Autotaxin has lysophospholipase D activity leading to tumor cell growth and motility by lysophosphatidic acid production. J Cell Biol 2002;158:227-233.

-6 Xiao YJ, Schwartz B, Washington M, Kennedy A, Webster K, Belinson J, Xu Y: Electrospray ionization mass spectrometry analysis of lysophospholipids in human ascitic fluids: comparison of the lysophospholipid contents in malignant vs nonmalignant ascitic fluids. Anal Biochem 2001;290:302-313.

-7 Xu Y, Shen Z, Wiper DW, Wu M, Morton RE, Elson P, Kennedy AW, Belinson J, Markman M, Casey G: Lysophosphatidic acid as a potential biomarker for ovarian and other gynecologic cancers. JAMA 1998;280:719-723.

8 Ren J, Xiao YJ, Singh LS, Zhao X, Zhao Z, Feng L, Rose TM, Prestwich GD, Xu Y: Lysophosphatidic acid is constitutively produced by human peritoneal mesothelial cells and enhances adhesion, migration, and invasion of ovarian cancer cells. Cancer Res 2006;66:3006-3014.

-9 Yu S, Murph MM, Lu Y, Liu S, Hall HS, Liu J, Stephens C, Fang X, Mills GB: Lysophosphatidic acid receptors determine tumorigenicity and aggressiveness of ovarian cancer cells. J Natl Cancer Inst 2008;100:16301642.

10 Blaho VA, Hla T: Regulation of mammalian physiology, development, and disease by the sphingosine 1-phosphate and lysophosphatidic acid receptors. Chem Rev 2011;111:6299-6320.

-11 Contos JJ, Ishii I, Chun J: Lysophosphatidic acid receptors. Mol Pharmacol 2000;58:1188-1196.

12 Chun J, Hla T, Lynch KR, Spiegel S, Moolenaar WH: International Union of Basic and Clinical Pharmacology. LXXVIII. Lysophospholipid receptor nomenclature. Pharmacol Rev 2010;62:579-587.

13 Bian D, Su S, Mahanivong C, Cheng RK, Han Q, Pan ZK, Sun P, Huang S: Lysophosphatidic Acid Stimulates Ovarian Cancer Cell Migration via a Ras-MEK Kinase 1 Pathway. Cancer Res 2004;64:4209-4217.

-14 Hong JH, Hwang ES, McManus MT, Amsterdam A, Tian Y, Kalmukova R, Mueller E, Benjamin T, Spiegelman BM, Sharp PA, Hopkins N, Yaffe MB: TAZ, a transcriptional modulator of mesenchymal stem cell differentiation. Science 2005;309:1074-1078.

15 Hong W, Guan KL: The YAP and TAZ transcription co-activators: key downstream effectors of the mammalian Hippo pathway. Semin Cell Dev Biol 2012;23:785-793. 


\section{Cellular Physiology and Biochemistry}

Cell Physiol Biochem 2013;32:253-263

\begin{tabular}{l|l}
\hline DOI: $10.1159 / 000354434$ & (c) 2013 S. Karger AG, Basel
\end{tabular}

Jeong et al.: Role of TAZ in LPA-Induced Cell Migration

16 Kanai F, Marignani PA, Sarbassova D, Yagi R, Hall RA, Donowitz M, Hisaminato A, Fujiwara T, Ito Y, Cantley LC, Yaffe MB: TAZ: a novel transcriptional co-activator regulated by interactions with 14-3-3 and PDZ domain proteins. EMBO J 2000;19:6778-6791.

17 Pan D: Hippo signaling in organ size control. Genes Dev 2007;21:886-897.

18 Zeng Q Hong W: The emerging role of the hippo pathway in cell contact inhibition, organ size control, and cancer development in mammals. Cancer Cell 2008;13:188-192.

19 Wang K, Degerny C, Xu M, Yang XJ: YAP, TAZ, and Yorkie: a conserved family of signal-responsive transcriptional coregulators in animal development and human disease. Biochem Cell Biol 2009;87:77-91.

20 Lei QY, Zhang H, Zhao B, Zha ZY, Bai F, Pei XH, Zhao S, Xiong Y, Guan KL: TAZ promotes cell proliferation and epithelial-mesenchymal transition and is inhibited by the hippo pathway. Mol Cell Biol 2008;28:24262436.

21 Zhao B, Ye X, Yu J, Li L, Li W, Li S, Yu J, Lin JD, Wang CY, Chinnaiyan AM, Lai ZC, Guan KL: TEAD mediates YAP-dependent gene induction and growth control. Genes Dev 2008;22:1962-1971.

-22 Chan SW, Lim CJ, Guo K, Ng CP, Lee I, Hunziker W, Zeng Q, Hong W: A role for TAZ in migration, invasion, and tumorigenesis of breast cancer cells. Cancer Res 2008;68:2592-2598.

-23 Yu FX, Zhao B, Panupinthu N, Jewell JL, Lian I, Wang LH, Zhao J, Yuan H, Tumaneng K, Li H, Fu XD, Mills GB, Guan KL: Regulation of the Hippo-YAP pathway by G-protein-coupled receptor signaling. Cell 2012;150:780-791.

24 Miller E, Yang J, DeRan M, Wu C, Su AI, Bonamy GM, Liu J, Peters EC, Wu X: Identification of serum-derived sphingosine-1-phosphate as a small molecule regulator of YAP. Chem Biol 2012;19:955-962.

25 Mo JS, Yu FX, Gong R, Brown JH, Guan KL: Regulation of the Hippo-YAP pathway by protease-activated receptors (PARs). Genes Dev 2012;26:2138-2143.

26 Stahle M, Veit C, Bachfischer U, Schierling K, Skripczynski B, Hall A, Gierschik P, Giehl K: Mechanisms in LPA-induced tumor cell migration: critical role of phosphorylated ERK. J Cell Sci 2003;116:3835-3846.

-27 Du J, Sun C, Hu Z, Yang Y, Zhu Y, Zheng D, Gu L, Lu X: Lysophosphatidic acid induces MDA-MB-231 breast cancer cells migration through activation of PI3K/PAK1/ERK signaling. PLoS One 2010;5:e15940.

28 Ohsawa S, Sato Y, Enomoto M, Nakamura M, Betsumiya A, Igaki T: Mitochondrial defect drives nonautonomous tumour progression through Hippo signalling in Drosophila. Nature 2012;490:547-551.

29 O'Neill E, Kolch W: Taming the Hippo: Raf-1 controls apoptosis by suppressing MST2/Hippo. Cell Cycle 2005;4:365-367.

-30 Cai H, Xu Y: The role of LPA and YAP signaling in long-term migration of human ovarian cancer cells. Cell Commun Signal 2013;11:31.

-31 Artemenko Y, Batsios P, Borleis J, Gagnon Z, Lee J, Rohlfs M, Sanseau D, Willard SS, Schleicher M, Devreotes PN: Tumor suppressor Hippo/MST1 kinase mediates chemotaxis by regulating spreading and adhesion. Proc Natl Acad Sci USA 2012;109:13632-13637.

-32 Hall CA, Wang R, Miao J, Oliva E, Shen X, Wheeler T, Hilsenbeck SG, Orsulic S, Goode S: Hippo pathway effector Yap is an ovarian cancer oncogene. Cancer Res 2010;70:8517-8525. 